\title{
Prodromal non-motor symptoms of Parkinson's disease
}

\author{
Clelia Pellicano \\ Dario Benincasa \\ Vincenzo Pisani \\ Francesca R Buttarelli \\ Morena Giovannelli \\ Francesco E Pontieri \\ Dipartimento di Scienze \\ Neurologiche, II Facoltà di Medicina \\ e Chirurgia; Università degli Studi di \\ Roma "La Sapienza”, Italy
}

Correspondence: Francesco E Pontieri Dipartimento di Scienze Neurologiche, II Facoltà di Medicina e Chirurgia, Università degli Studi di Roma "La Sapienza",

UOC Neurologia-Azienda Ospedaliera

Sant'Andrea,Via di Grottarossa,

I035-00 I 89 Roma, Italy

TeL +390633775579

Fax +390633775900

Email francesco.pontieri@

ospedalesantandrea.it

\begin{abstract}
The motor symptoms of Parkinson's disease (PD), bradykinesia, muscular rigidity, and tremor depend upon degeneration of the dopaminergic neurons in the substantia nigra pars compacta. Recent neuropathological studies show that the Lewy bodies, the intraneuronal landmark of PD, accumulate in several neuronal cell types in the brain. An ascending gradient of pathological involvement, from the medulla oblongata to neocortical areas has been reported. Thus the original view of PD as a disease characterized by selective damage of the dopaminergic neurons in the mesencephalon should be updated into the concept of a severe multisystemic neurodegenerative disorder. Additionally, the neuropathological alterations outside the substantia nigra are soundly correlated with the non-motor symptoms of PD. As a result of these findings, interest is growing in the identification of prodromal non-motor symptoms of PD. Indeed, data from the literature suggest that autonomic disturbances, olfactory dysfunctions, depression and sleep disorders (in particular REM-sleep behavior disorder) may represent prodromal non-motor symptoms of PD. Several tests are available to detect most of these symptoms. Thus, the identification of prodromal non-motor symptoms may contribute to the precocious diagnosis of PD, and might be useful in the future to test the efficacy of neuroprotective agents.
\end{abstract}

Keywords: Parkinson's disease, non-motor symptoms, autonomic dysfunction, olfactory dysfunction, depression, RBD

\section{Introduction}

Parkinson's disease (PD) is a common neurodegenerative disorder characterized by bradykinesia, muscular rigidity, and tremor (Klockgether 2004). Among the three cardinal symptoms, bradykinesia is the most important and mainly contributes to the disability associated with PD progression. The term bradykinesia refers to the slowness and poverty of voluntary and involuntary movements characteristic of parkinsonism. Muscular rigidity, defined as the increased resistance of a joint to passive movements, only minimally contributes to the impairment experienced by parkinsonian patients. The subjective correlate of rigidity is a feeling of stiffness and reduced ability to relax limb and trunk muscles. Tremor is the most conspicuous of the cardinal symptoms of PD, and gave rise to the initial name of the disease, shaking palsy, by James Parkinson in 1817. Parkinsonian tremor usually occurs during rest, and typically affects the upper limbs, but may also occur in the legs and less frequently in the head. Although resting tremor is not necessarily disabling, many patients suffer considerably from it, not least because tremor stigmatizes them as parkinsonian subjects.

The cardinal motor symptoms of PD depend upon progressive degeneration of the dopamine-containing neurons in the substantia nigra pars compacta (Hughes et al 1992). The nigrostriatal dopaminergic damage can be monitored by functional neuroimaging techniques, such as positron emission tomography (PET) or single photon emission tomography (SPECT) (Eckert and Eidelberg 2005). Such methods contributed to establish 
that approximately 50\% reduction in dopaminergic nigrostriatal cells is required before clinical expression of the motor symptoms (Schwarz et al 2000; Marek et al 2001).

Therapy of PD is, at present, symptomatic, aimed at ameliorating clinical symptoms (Rascol et al 2002). Available drugs include levodopa, dopamine receptor agonists, anticholinergics, and antiglutamatergics. A more complete therapeutic approach to PD, however, should also be aimed at neuroprotection (slowing the progression of neuronal degeneration), neurorescue (normalizing injured neurons that are not yet dead), and neurorestoration (increasing the number of neurons by directly implanting new ones or causing existing cells to divide). Although functional neuroimaging data support the potential neuroprotective effect of some dopamine receptor agonists in PD (Parkinson Study Group 2000; Whone et al 2003), the development of effective neuroprotective strategies is still limited by difficulties in making the correct diagnosis early during the course of illness.

Recent neuropathological data indicate that the pathological landmark of PD, the Lewy bodies, can be identified in several neuronal populations other than the dopaminergic mesencephalic ones (Braak et al 2003). Following the ascending gradient of neuronal involvement throughout disease progression, 6 neuropathological stages of PD have been identified (Braak et al 2004): in stages 1 and 2, defined as "presymptomatic stages", the Lewy bodies are confined to the anterior olfactory nucleus, olfactory bulb, dorsal motor nucleus of the glossopharyngeal and vagal nerves, locus coeruleus, and reticular formation. In stages 3 and 4 ("intermediate stages"), the neuropathological damage extends to the substantia nigra pars compacta, other mesencephalic nuclei, the prosencephalon and meso-allocortical regions; during these stages, the motor symptoms develop and progressively worsen. Eventually, in stages 5 and 6 ("advanced stage" of PD), neocortical, prefrontal, and associative cortices are pathologically involved; in these latter stages, severe motor disturbances are accompanied by cognitive and behavioral symptoms. As a result of these neuropathological findings, the original description of $\mathrm{PD}$ as due to the selective damage of dopaminergic neurons in the mesencephalon should be updated into the concept of a severe multisystemic neurodegenerative disorder, whose clinical symptoms reflect the progression of the pathological involvement from the medulla oblongata to neocortical areas.

One of the most intriguing aspects of the studies by Braak et al $(2003,2004)$ is that the neuropathological alterations outside the substantia nigra are soundly correlated with some non-motor symptoms of PD. Thus, the identification of early non-motor symptoms of PD and the application of instrumental methodologies may contribute to making the correct diagnosis of PD during the pre-motor stages. To this end, the identification of genetic mutations responsible for hereditary forms of PD (Bonifati et al 2004) plays a crucial role, as it allows study of mutation carriers before the onset of the motor symptoms.

Based on the localization of Lewy bodies during the presymptomatic stages of PD, the following non-motor symptoms may play a role as prodromal markers of the disease:

- Autonomic disturbances

- Olfactory dysfunctions

- Psychiatric symptoms

- Sleep disorders, in particular REM sleep behavior disorder (RBD)

\section{Prodromal non-motor symptoms of PD}

\section{Autonomic disturbances}

Dysautonomia is a key characteristic of multiple system atrophy (MSA), but occurs with varying severity in PD (Chauduri 2001). The pathophysiology of dysautonomia in PD is complex and includes degeneration and dysfunction of autonomic nuclei such as the dorsal vagal nucleus, the nucleus ambiguus, and other medullary nuclei which exert differential control on the sympathetic preganglionic neurons via descending pathways (Benarroch 1999). Additionally, degeneration of cholinergic, monoaminergic and serotonergic nuclei may cause abnormalities of modulatory effects within the central autonomic network (Benarroch 1999).

Subtle autonomic disturbances that can at least partly be related to the degeneration of the vagal nerve are an early and frequent sign in PD (Micieli et al 2003). Almost all PD patients suffer from constipation (Magerkurth et al 2005). An epidemiological study of 6790 men followed prospectively in the Honolulu Heart Program showed that men with less than one bowel movement per day had a 4.5-fold excess risk of developing PD versus men with more than two bowel movements per day (Abbott et al 2001). These observations are compatible with the view that constipation is part of an early PD process. Nevertheless, such gastrointestinal symptoms are poorly specific in nature, besides being often aggravated by drugs such as tricyclic antidepressants.

Although troublesome dysautonomia is only thought to complicate advanced disease, cardiac meta-iodobenzylguanidine (MIGB) imaging challenges this view by showing early cardiac sympathetic denervation in PD and not multiple 
system atrophy (Goldstein et al 2000). Cardiac sympathetic denervation has also been linked to genetic forms of PD with alpha-synuclein mutations (Singleton et al 2004).

Autonomic function in PD can be objectively assessed by several validated methods, including quantitative sudomotor axon reflex test, urodynamic studies, defecating proctography, tilt test, sympathetic skin response, and pupil function test with pilocarpine or phenylepinephrine (Bannister and Mathias 1999). In cases where distinction from MSA is needed, tests such as urethral sphincter electromyography, plasma norepinephrine concentration in response to head-up tilting, and clonidine-induced growth hormone challenge test may be useful (Bannister and Mathias 1999).

\section{Olfactory dysfunctions}

Olfactory disorders occur frequently in PD. More than 50\% of patients suffering PD experience anosmia, 35\% of patients have severe hyposmia and $14 \%$ of patients moderate hyposmia (Hawkes et al 1997; Muller et al 2002). The olfactory deficit in PD shows above all an increase of olfactory threshold, and minor alterations of identificative and discriminative functions (Tissingh et al 2001). The olfactory dysfunctions in PD depend on damage of the dopaminergic neurons in the olfactory bulb and olfactory nuclei. However, olfactory deficit is not responsive to antiparkinsonian drugs and is not related to the severity of motor symptoms (Doty et al 1988, 1992; Tissingh et al 2001). Such apparent discrepancy may depend on the precocious damage of the olfactory system in PD, which makes olfactory dysfunction already advanced at the time of onset of motor symptoms, ie, when the clinical diagnosis of PD is made.

Several lines of evidence suggest that olfactory dysfunction is a potential pre-motor marker of PD. Ansari and Johnson (1975) first suggested the association between olfactory dysfunction and the development of PD. Subsequently, Doty et al (1988, 1992), Muller et al (2002), and Ponsen et al (2004) indicated hyposmia as a prodromal marker of the disease. Studies on healthy first degree relatives of parkinsonians in families with hereditary or sporadic PD confirmed that olfactory dysfunctions may be a pre-motor marker of the disease: indeed, olfactory dysfunctions have been identified in $41 \%$ of healthy subjects in families with autosomal dominant PD (Markopoulou et al 1997), and other studies reported that olfactory dysfunction is associated with an increased risk of developing PD among first degree relatives of sporadic PD patients (Wolters et al 2000; Ponsen et al 2004).

Further data suggest that olfactory function may be a potentially useful test also to distinguish PD from atypical parkinsonism. In a controlled but unblinded study using the University of Pennsylvania Smell Identification Test (UPSIT), patients with progressive supranuclear palsy (PSP) had similar olfaction to normal controls, but differed from patients with PD (Doty et al 1993). Using the same method, Wenning et al (1995) reported normal olfactory scores in PSP and corticobasal ganglionic degeneration, and significantly worse scores in PD patients. Interestingly, patients with MSA scored worse than control, but significantly better than PD. Finally, in a prospective study using the "sniffing sticks", all PD patients had moderate to severe hyposmia or anosmia, while patients with other parkinsonisms or essential tremor (ET) had normal or only mild to moderate hyposmia (Muller et al 2002).

\section{Psychiatric symptoms}

Depression is extremely frequent in PD, occurring in up to $45 \%$ of cases (Burns 2002). Depression is not necessarily related to the severity of motor symptoms of PD, and is often misdiagnosed since hypomimia and reduction of voluntary movements are common to both PD and pure depression. Therefore, the identification of depression in PD patients is essentially based upon subjective perception of depressive symptoms such as feeling of incapability, reduced reaction to emotional stimuli, and inability to experience pleasure from things and events (anhedonia).

The pathophysiology of depression in PD is rather complex, being related to damage of the noradrenergic, serotonergic and dopaminergic pathways in the brain (Doder et al 2000; Remy et al 2005). Indeed, the pattern of depressive symptoms in PD appears different from that of pure depression. Typical signs of depression in PD are a raised degree of dysphoria and irritability, pessimism about future, with low levels of inadequacy and sense of guilt (Remy et al 2005).

Interest in depression in PD has increased recently not only for the need of pharmacological treatment, but also for its potential role as a pre-motor marker of the disease. In a case-control study, the occurrence of depression in the last 5 years was significantly linked to future development of motor symptoms of PD (Shiba et al 2000). Similar findings were drawn from a cohort study (Nilsson et al 2001). A longitudinal study showed the increased risk of developing PD in depressed subjects as compared to not depressed ones (Schurmann et al 2002). Finally, Lauterbach et al (2004) reported that patients with PD more often had a primary (preceding the onset of PD) diagnosis of single phobia and a secondary atypical depression than did those with dystonia. In conclusion, although the pathophysiological and 
neurochemical features linking the two disorders are not completely known, the correlation between depression and PD is consolidated to such a point that depression can be considered a non-motor sign of $\mathrm{PD}$, potentially useful in the pre-motor stages of the disease.

Anxiety is also common in PD, and may represent a premotor risk factor (Shiba et al 2000; Weisskopf et al 2003). Anxiety can be present as panic attacks, phobias, or generalized anxiety disorder, and can be related to drug-induced motor fluctuations in PD.

\section{Sleep disorders}

Virtually all PD patients develop sleep disruption, and there is evidence that the process usually begins early in the course of the disease (Chaudhuri 2003). Sleep disturbance in PD has a multifactorial etiology, but pathological degeneration of central sleep regulation centers in the brainstem and thalamocortical pathways is probably important (Saper et al 2001).

RBD is a specific disorder during the REM phase of sleep (Gagnon et al 2002), characterized by sudden motorbehavioral episodes of violent nature, associated with attitudes of defense from a threat and vocalizations. The oneiric experience lived by the patient, which almost always has a threatening and unpleasant content, is closely reflected in his movements. Sometimes, the motor features are directed towards others.

Sleep disorders, and RBD in particular, are often seen in synucleinopathies such as PD, MSA, and diffuse Lewy body dementia (Shenk et al 1996; Olson et al 2000). The prevalence of RBD in PD is estimated at between 15\% and $33 \%$, increasing to $58 \%$ if one takes into account subclinical RBD (Shenk et al 1996). Some reports show that RBD evolves together with the motor symptoms in PD, and also that RBD can precede the onset of motor symptoms. In this respect, two epidemiological studies proved that $\mathrm{PD}$ develops in 40\%-50\% of subjects suffering RBD (Schenk et al 1996; Olson et al 2000). Moreover, the evidence of dopaminergic nigrostriatal damage in RBD, as measured by SPECT (Eisensehr et al 2000), suggests that RBD can represent an early symptom of PD, in particular when associated with olfactory dysfunction (Stiasny-Kolster et al 2005).

In addition to $\mathrm{RBD}$, recent data suggest that excessive daytime sleepiness may also be a pre-motor marker of PD (Abbott et al 2005). It is important to recognize excessive daytime sleepiness, as it can substantially affect quality of life in PD. A combination of the disease process, the effect of nocturnal sleep deprivation, and antiparkinsonian drugs is probably causative and, in some patients, excessive daytime sleepiness has been linked to the development of suddenonset sleep (Tracik and Ebersbach 2001).

\section{Biomarkers}

Neuropathological and clinical findings suggest, therefore, that PD is a multisystemic neurodegenerative disorder, and that not-motor symptoms may play a role not only in determining functional impairment, but also as early markers of the disease.

Although autonomic or olfactory dysfunctions, psychiatric symptoms and sleep disorders may represent prodromal markers of PD, it should be pointed out that none of these symptoms is specific to PD, since they all may occur as idiopathic disorders or during the course of other neurodegenerative diseases, such as Alzheimer's dementia (Idiaquez et al 2002; Hoyt 2005; Burgut et al 2006; Hawkes 2006). Thus, the identification of pre-motor signs in subjects at risk to develop PD needs to be associated with instrumental evidence of the damage to dopamine nigrostriatal pathway in order to make a correct presymptomatic diagnosis of the disease.

\section{Functional neuroimaging}

One of the goals of functional neuroimaging is the presymptomatic detection of patients, and there are now several publications to suggest this may be possible. For example, patients with hemi-Parkinson's disease showed reduced uptake of the ipsilateral as well as the expected contralateral side (Schwarz et al 2000), twin studies have shown a dopaminergic deficit in asymptomatic twins of patients with PD (Burns et al 1992; Holthoff et al 1994; Lahinen et al 2000), and dopaminergic dysfunction has been shown in people after taking 1-methyl-4-phenyl-1,2,3,6-tetrahydropyridine prior to the onset of motor symptoms (Calne et al 1985). However, one unresolved issue with these studies is whether subjects with preclinical imaging abnormalities actually go on to develop PD, although data from one of the twin studies suggest that at least some do (Piccini et al 1997). Moreover, the high cost of these methodologies prevents their application to the screening on wide populations of at risk subjects. Thus, interest is growing in the characterization of other tools that can contribute to the early identification of the damage of dopaminergic neurons in PD.

\section{Transcranial sonography}

In 1995, Becker et al reported a highly characteristic enlargement of echogenic signal (hyperechogenicity) of the substantia nigra in idiopathic PD. Since then, a number of 
studies has been performed indicating that this method is a valuable tool for the differential diagnosis of PD, which even allows the identification of subjects at risk for nigrostriatal dysfunction. Evidence that this method is indeed valid comes from the following findings: (i) several groups independently demonstrated that the hyperechogenicity of the substantia nigra is a characteristic ultrasound finding in more than $90 \%$ of PD patients (Berg et al 2001; Walter et al 2002; Zedde et al 2005); (ii) the reproducibility is high, with very low inter rater variation (Berg et al 1999; 2001); (iii) prospective studies with two independent sonographers/raters, of whom at least one was blinded to the clinical data of patients, indicate a high predictive value of an association between hyperechogenicity of the substantia nigra and nigrostriatal impairment (Berg et al 1999; Walter et al 2003); (iv) substantia nigra hyperechogenicity indeed reflects an alteration linked to nigrostriatal impairment, since it is larger contralateral to the clinically more affected side (Berg et al 2001), it is present in PD but not ET, and only very rarely in atypical parkinsonian syndromes, such as striatonigral degeneration and PSP (Walters et al 2003; Niehaus et al 2004; Behnke et al 2005), and can be visualized in healthy subjects showing mild impairment of the nigrostriatal system (Berg et al 1999); (v) lastly, substantia nigra hyperechogenicity appears associated with the reduction of striatal ${ }^{18} \mathrm{~F}$-dopa uptake in healthy subjects (Berg et al 1999, 2002) as well as in asymptomatic parkin mutation carriers (Walter et al 2004), and in subjects with olfactory dysfunction (Sommer et al 2004). Thus transcranial sonography is a potential diagnostic tool in PD. It is attractive because it is a non-invasive, low risk test. However, more research needs to be performed to better determine if it is useful in the setting of diagnostic ambiguity early in the course of parkinsonism. Similarly, it remains to be established how well transcranial sonography performs in distinguishing PD and atypical parkinsonian syndromes, MSA in particular, and if it can be reliably used by multiple raters throughout the movement disorders centers.

\section{Blood tests}

PD is thought to be due, at least in part, to oxidative stress (Jenner 2003), and considerable effort has been spent in the search of a marker of this process. For example, patients with PD show a selective reduction in mitochondrial complex I in the substantia nigra (Shapira et al 1990), as well as in their platelets (Parker et al 1989). The concentrations of several other potential markers of oxidative stress have been measured in the blood, such as malondialdehyde, superoxide radicals (Ilic et al 1999), the coenzyme Q10 redox ratio (Gotz et al 2000), 8-hydroxy-2'-deoxyguanosine from oxidized DNA, and 8-hydroxyguanosine from RNA oxidation (Kikuchi et al 2002; Abe et al 2003). The levels tend to be abnormal in PD compared with control groups, providing valuable insight into the nature of oxidative stress, but none is sufficiently robust to be useful as a diagnostic marker of the disease process in the clinical practice. A similar situation exists if instead the levels of protective enzymes systems are compared, such as glutathione reductase, or copper and zinc superoxide dismutase (Ilic et al 1999).

An alternative approach may arise from studies dealing with peripheral dopamine system in PD. Studies on peripheral blood mononuclear cells have shown the reduction of intracellular dopamine concentrations (Caronti et al 1999), and tyrosine hydroxylase and dopamine transporter immunoreactivities (Caronti et al 1999; 2001). There is also evidence on the modulation of the expression of dopamine receptor mRNAs in the same cells (Nagai et al 1996; Barbanti et al 1999). Although these findings are very promising, further studies are necessary before considering such methods useful for the differential diagnosis of PD as well as for the identification of at risk subjects.

\section{References}

Abbott RD, Petrovitch H, White LR, et al. 2001. Frequency of bowel movements and the future risk of Parkinson's disease. Neurology, 57:456-62.

Abbott RD, Ross GW, White LR, et al. 2005. Excessive daytime sleepiness and subsequent development of Parkinson's disease. Neurology, 65:1442-6.

Abe T, Isobe C, Murata T, et al. 2003. Alteration of 8-hydroxyguanosine concentrations in the cerebrospinal fluid and serum from patients with Parkinson's disease. Neurosci Lett, 336:105-8.

Ansari KA, Johnson A. 1975. Olfactory dysfunction in patients with Parkinson's disease. J Chronic Dis, 28:493-7.

Bannister R, Mathias CJ. 1999. Clinical features and evaluation of primary chronic autonomic failure syndromes. In: Mathias CJ, Bannister R (eds). Autonomic failure. 4th ed. Oxford: Oxford Univ. Pr. pp. 307-16.

Barbanti P, Fabbrini G, Ricci A, et al. 1999. Increased expression of dopamine receptors on lymphocytes in Parkinson's disease. Mov Disord, 14:764-77.

Becker G, Seufert J, Bogdahn U, et al. 1995. Degeneration of substantia nigra in chronic Parkinson's disease visualized by transcranial colorcoded real-time sonography. Neurology, 45:182-4.

Behnke S, Berg D, Naumann M, et al. 2005. Differentiation of Parkinson“s disease and atypical parkinsonian syndromes by transcranial ultrasound. J Neurol Neurosurg Psychiatry, 76:423-5.

Benarroch EE. 1999. Central neurotransmitters and neuromodulators in cardiovascular regulation. In: Mathias CJ, Bannister R (eds). Autonomic failure. 4th ed.Oxford: Oxford Univ. Pr. pp. 37-44.

Berg D, Becker G, Zeiler B, et al. 1999. Vulnerability of the nigrostriatal system as detected by transcranial ultrasound. Neurology, 53:1026-31.

Berg D, Siefker C, Becker G. 2001. Echogenicity of the substantia nigra in Parkinson's disease and its relation to clinical findings. J Neurol, 8:684-9.

Berg D, Roggendorf W, Schroder U, et al. 2002. Echogenicity of the substantia nigra:association with increased iron content and marker for susceptibility to nigrostriatal injury. Arch Neurol, 59:999-1005. 
Bonifati V, Oostra BA, Heutink P. 2004. Unraveling the pathogenesis of Parkinson's disease - the contribution of monogenic forms. Cell Mol Life Sci, 61:1729-50.

Braak H, Del Tredici K, Rub U, et al. 2003. Staging of brain pathology related to sporadic Parkinson's disease. Neurobiol Aging, 24:197-211.

Braak H, Ghebremedhin E, Rub U, et al. 2004. Stages in the development of Parkinson's disease-related pathology. Cell Tissue Res, 318:121-34.

Burgut FT, Benaur M, Hencliffe C. 2006. Late-life depression:a neuropsychiatric approach. Expert Rev Neurother, 6:65-72.

Burns DJ, Mark MH, Playford ED, et al. 1992. Parkinson's disease in twins studied with 18F-dopa and positron emission tomography. Neurology, 42:1894-900.

Burns DJ. 2002. Beyond the iron mask:towards better recognition and treatment of depression associated with Parkinson's disease. Mov Disord, 17:445-54.

Calne DB, Langston JW, Martin WR, et al. 1985. Positron emission tomography after MPTP:observations relating to the cause of Parkinson's disease. Nature, 317:246-8.

Caronti B, Tanda G, Calderaro C, et al. 1999. Reduced dopamine in peripheral blood lymphocytes in Parkinson's disease. Neuroreport, 10:2907-10.

Caronti B, Antonini G, Calderaro C, et al. 2001. Dopamine transporter immunoreactivity in peripheral blood lymphocytes in Parkinson's disease. J Neural Transm, 108:803-7.

Chaudhuri KR. 2001. Autonomic dysfunction in movement disorders. Curr Opin Neurol, 14:505-11.

Chaudhuri KR. 2003. Noctural symptom complex in PD and its management. Neurology, 61 (Suppl 3):S17-S23.

Doder M, Rabiner EA, Turjanski N, et al. 2000. Brain serotonin 5HT, A receptors in Parkinson's disease with and without depression measured by positron emission tomography with ${ }^{11} \mathrm{C}-\mathrm{WAY} 10635$. Mov Disord, 15:213.

Doty RL, Deems DA, Stellar S. 1988. Olfactory dysfunction in parkinsonism:a general deficit unrelated to neurological signs, disease stage, or disease duration. Neurology, 39:1237-44.

Doty RL, Stern MB, Pfeiffer C, et al. 1992. Bilateral olfactory dysfunction in early stage treated and untreated idiopathic Parkinson's disease. $J$ Neurol Neurosurg Psychiatry, 55:138-42.

Doty RL, Golbe LI, McKeown DA, et al. 1993. Olfactory testing differentiates between progressive supranuclear palsy and idiopathic Parkinson's disease. Neurology, 43:962-5.

Eisensehr I, Linke R, Noatchar S, et al. 2000. Reduced striatal dopamine transporter in idiopathic rapid eye movement sleep behavior disorder:comparison with Parkinson's disease and controls. Brain, 123:1155-60.

Eckert T, Eidelberg D. 2005. Neuroimaging and therapeutics in movement disorders. NeuroRx, 2:361-71.

Gagnon JF, Bedard MA, Fantini ML, et al. 2002. REM sleep behavior disorder and REM sleep without atonia in Parkinson's disease. Neurology, 59:585-9.

Goldstein DS, Holmes C, Li ST, et al. 2000. Cardiac sympathetic denervation in Parkinson's disease. Ann Intern Med, 133:338-47.

Gotz ME, Gerstner A, Harth R, et al. 2000. Altered redox state of platelet coenzyme Q10 in Parkinson's disease. J Neural Transm, 107:41-8.

Hawkes CH, Shephard BC, Daniel SE. 1997. Olfactory dysfunction in Parkinson's disease. J Neurol Neurosurg Psychiatry, 62:436-46.

Hawkes CH. 2006. Olfaction in neurodegenerative disorders. Acta Otorhinolaryngol, 63:133-51.

Holthoff VA, Vieregge P, Kessler J, et al. 1994. Discordant twins with Parkinson's disease:positron emission tomography and early signs of impaired cognitive circuits. Ann Neurol, 36:176-82.

Hoyt BD. 2005. Sleep in patients with neurologic and psychiatric disorders. Prim Care, 32:535-48.

Hughes AJ, Daniel SE, Kilford L, et al. 1992. Accuracy of clinical diagnosis of idiopathic Parkinson's disease:a clinico-pathological study of 100 cases. J Neurol Neurosurg Psychiatry, 55:181-4.

Idiaquez J, Sandoval E, Seguel A. 2002. Association between neuropsychiatric and autonomic dysfunction in Alzheimer's disease. Clin Auton Res, 12:43-6.
Ilic TV, Jovanovic M, Jovicic A, et al. 1999. Oxidative stress indicators are elevated in de novo Parkinson's disease patients. Funct Neurol, $14: 141-147$

Jenner P. 2003. Oxidative stress in Parkinson's disease. Ann Neurol, 53 (Suppl 3):S26-S36.

Kikuchi A, Takeda A, Onodera H, et al. 2002. Systemic increase of oxidative nucleic acid damage in Parkinson's disease and multiple system atrophy. Neurobiol Dis, 9:244-248.

Klockgether T. 2004. Parkinson's disease:clinical aspects. Cell Tissue Res, 318:115-20.

Lauterbach EC, Freeman A, Vogel RL. 2004. Differential DSM-III psychiatric disorder prevalence profiles in dystonia and Parkinson's disease. $J$ Neuropsychiatry Clin Neurosci, 16:29-36.

Lahinen A, Ruottinen H, Rinne OJ, et al. 2000. Risk for Parkinson's disease: twin studies for the detection of asymptomatic subjects using [18F]6fluorodopa PET. J Neurol, 247 (Suppl 2):II110-II113.

Magerkurth C, Schnitzer R, Braune S. 2005. Symptoms of autonomic failure in Parkinson's disease:prevalence and impact on daily life. Clin Auton Res, 15:76-82.

Marek K, Innis R, Van Dyck C, et al. 2001. [123I]-beta-CIT-SPECT imaging assessment of the rate of Parkinson's disease progression. Neurology, 57:2089-94

Markopoulou K, Larsen KW, Wszolek EK, et al. 1997. Olfactory dysfunction in familial parkinsonism. Neurology, 49:1262-7.

Micieli G, Tosi P, Marcheselli S, et al. 2003. Autonomic dysfunction in Parkinson's disease. Neurol Sci, 24 (Suppl 1):S32-S34.

Muller A, Reichmann H, Livermore A, et al. 2002. Olfactory function in idiopathic Parkinson's disease (IPD):results from cross-sectional studies in IPD patients and long-term follow-up of de novo-IPD patients. $J$ Neural Transm, 109:805-11.

Nagai Y, Ueno S, Saeki Y, et al. 1996. Decrease of the D3 dopamine receptor mRNA expression in lymphocytes from patients with Parkinson's disease. Neurology, 46:791-5.

Niehaus L, Savyer N, Weber U, et al. 2004. Brain parenchyma sonography in patients with essential tremor and Parkinson's disease. Cerebrovasc Dis, 17(Suppl 4):3.

Nilsson FM, Kessig LV, Bolwig TG. 2001. Increased risk of developing Parkinson's disease for patients with major affective disorders. Acta Psychiatr Scand, 104:380-6.

Olson EJ, Boeve BF, Silber MH. 2000. Rapid eye movement sleep behavior disorder:demographic, clinical and laboratory findings in 93 cases. Brain, 123:388-93.

Parker WD Jr, Boyson SJ, Parks JK. 1989. Abnormalities of the electron transport chain in idiopathic Parkinson's disease. Ann Neurol, 26:719-23.

Parkinson Study Group. 2000. Pramipexole versus levodopa as initial treatment for Parkinson disease:a randomized controlled trial. JAMA, 284:1931-8.

Piccini P, Morrish PK, Turjanski N, et al. 1997. Dopaminergic function in familial Parkinson's disease: a clinical and 18F-dopa positron emission tomography study. Ann Neurol, 41:222-9.

Ponsen MM, Stoffers D, Booij J, et al. 2004. Idiopathic hyposmia as a preclinical sign of Parkinson's disease. Ann Neurol, 56:173-81.

Rascol O, Goetz C, Koller W, et al. 2002. Treatment interventions for Parkinson's disease:an evidence based assessment. Lancet, 359:1589-98.

Remy P, Doder M, Lees A, et al. 2005. Depression in Parkinson's disease: loss of dopamine and noradrenaline innervation in the limbic system. Brain, 128:1314-22.

Saper C, Chou TC, Scammell TE. 2001. The sleep switch:hypothalamic control of sleep and wakefulness. Trends Neurosci, 24:726-31.

Schenk CH, Bundlie SR, Mahowald MW. 1996. Delayed emergence of a parkinsonian disorder in $38 \%$ of 29 older men initially diagnosed with idiopathic rapid eye movement sleep behavior disorder. Neurology, 46:388-93.

Schurmann AG, Van Den Akker H, Ensinck KTJL, et al. 2002. Increased risk of Parkinson's disease after depression:a retrospective cohort study. Neurology, 58:1501-4. 
Schwarz J, Linke R, Kerner M, et al. 2000. Striatal dopamine transporter binding assessed by $\left[{ }^{123} \mathrm{I}\right] \mathrm{IPT}$ and single photon emission computed tomography in patients with early Parkinson's disease: implications for a preclinical diagnosis. Arch Neurol, 57:205-8.

Shapira AH, Cooper JM, Dexter D, et al. 1990. Mitochondrial complex I deficiency in Parkinson's disease. J Neurochem 54:823-7.

Shiba M, Bower JH, Maraganore DM, et al. 2000. Anxiety disorders and depressive disorders preceding Parkinson's disease:a case-control study. Mov Disord, 15:669-77.

Singleton A, Gwinn-Hardy K, Sharabi Y. 2004. Association between cardiac denervation and parkinsonism caused by alpha-synuclein gene triplication. Brain, 127:768-72.

Sommer U, Hummel T, Cormann K, et al. 2004. Detection of presymptomatic Parkinson's disease:combinating smell tests, transcranial sonography, and SPECT. Mov Disord, 19:1196-202.

Stiasny-Kolster K, Doerr Y, Moller JC, et al. 2005. Combination of idiopathic REM sleep behaviour disorder and olfactory dysfunction as possible indicator for alpha-synucleinopathy demonstrated by dopamine transporter FP-CIT-SPECT. Brain, 128:126-37.

Tissingh G, Berendse HW, Bergmans P, et al. 2001. Loss of olfaction in de novo and treated Parkinson's disease:possible implications for early diagnosis. Mov Disord, 16:41-6.

Tracik F, Ebersbach G. 2001. Sudden daytime sleep onset in Parkinson's disease:polysomnographic recordings. Mov Disord, 16:500-6.

Walter U, Wittstock M, Benecke R, et al. 2002. Substantia nigra hecogenicity is normal in non-extrapyramidal cerebral disorders but increased in Parkinson's disease. J Neural Transm, 109:191-6.

Walter U, Niehaus L, Probst T, et al. 2003. Brain parenchyma sonography discriminates Parkinson's disease and atypical parkinsonian syndromes. Neurology, 60:74-7.

Walter U, Klein C, Hilker R, et al. 2004. Deep brain parenchyma sonography detects preclinical parkinsonism. Mov Disord, 19:1445-9.

Weisskopf MG, Chen H, Schwarzchild MA, et al. 2003. Prospective study of phobic anxiety and risk of Parkinson's disease. Mov Disord, 18:646-51.

Wenning GK, Shepard B, Hawkes C, et al. 1995. Olfactory function in atypical parkinsonian syndromes. Acta Neurol Scand, 91:247-50.

Whone AL, Watts RL, Stoessl AJ, et al. 2003. Slower progression of Parkinson's disease with ropinirole versus levodopa:the REAL-PET study. Ann Neurol, 54:93-101.

Wolters EC, Francot C, Bergmans P, et al. 2000. Preclinical (premotor) Parkinson's disease. J Neurol, 247 (suppl. 2):II103-9.

Zedde M, Manca A, Baule G, et al. 2005. Brain parenchyma sonography (BPS) of substantia nigra (SN) in Parkinson's disease. Parkinsonism \& Rel Dis, 11(Suppl 2):222. 
